high or low mating rates may have reduced fitness ${ }^{3}$, but such analyses must be treated with caution. Females are expected to be adapted to the number of matings they experience ${ }^{4}$, even if this is a compromise between their optimal mating rate and that which males attempt to impose.

More significantly, most of the costs to females that Gavrilets sees as driving sexual conflict (such as predation, sensory exploitation, different costs of mating or seminal fluid toxicity $)^{1}$ are paid as a result of matings per se, and hence can only be prevented if matings themselves are avoided. However, if incompatibilities prevent mating, then males are expected to be able to determine rapidly whether a particular female is compatible. Hence incompatibilities that prevent mating between particular male and female phenotypes will simply result in males only attempting to mate with females with whom they are compatible, with no reduction in the number of matings or attempted matings to which each female is subjected.

This suggests that the potential for sexual conflict to promote divergence may be limited to species without the possibility of pre-copulatory mate choice, but in which there are still costs to being compatible with too many males. There may be examples of such species - for instance, broadcast spawners may suffer from polyspermy but such cases are likely to be rare.

Although we question the generality of Gavrilets' model ${ }^{1}$, it does indicate the potential for the different priorities of males and females to drive evolution of reproductive isolation. Perhaps rather than lumping so much biology together under the title 'sexual conflict', we need to consider the specific processes involved.

Tom Tregenza, Roger K. Butlin, Nina Wedell School of Biology, University of Leeds,

Leeds LS2 9JT, UK

e-mail: gentbt@leeds.ac.uk

1. Gavrilets, S. Nature 403, 886-889 (2000).

2. Parker, G. A. \& Partridge, L. Phil. Trans. R. Soc. Lond. B 353 , 261-274 (1998).

. Arnqvist, G. \& Nilson, T. Anim. Behav. (in the press).

4. Holland, B. \& Rice, W. R. Proc. Natl Acad. Sci. USA 96,

5083-5088 (1999).

Gavrilets replies - Tregenza et al. maintain that my model's predictions ${ }^{1}$ run counter to the model of Parker and Partridge ${ }^{2}$, but it is not straightforward to compare these two classes of model because of their inherent differences.

First, my model considers genetic divergence of allopatric populations, whereas the models developed in ref. 2 describe the evolutionary consequences of a secondary contact between populations that have already diverged: that is, they model reinforcement of reproductive isolation rather than its emergence. Second, Parker and Partridge ${ }^{2}$ use an evolutionarily stable strategy framework with a set of fixed evolutionary options, whereas I model continuous coevolution of the sexes, during which evolutionary options are continuously changing.

In spite of these differences and contrary to the claim of Tregenza et al., there is no contradiction in the predictions of both types of model. Parker and Partridge propose that mating conflict could be either a hindrance to isolation if 'male-win' scenarios prevail, or a facilitator if females tend to win - exactly as predicted by my model if coevolution is restricted. However, my model takes an additional step by considering the possibility of continuous coevolution of the sexes. In this case the prediction is that neither sex will win the sexual conflict, but rather that there will be a dynamic coevolutionary compromise.

Tregenza et al. question the generality of my model's assumption that females have an intermediate optimum mating rate, as well as the specific (quadratic) function I used to model the relation between a female's fitness and her mating rate: in fact, the model is well supported by insect ${ }^{3}$ and other data ${ }^{4,5}$, and the idea that excessive mating rates are bad for females is the essence of sexual conflict.

Inadequate mating rates are also detrimental ${ }^{6}$. Thus, with sexual conflict the optimum mating rate must be intermediate. As for the shape of the relation between female mating costs and mating rate, more data are indeed necessary. However, I do not anticipate that using functions more complicated than a quadratic one will affect my main conclusions ${ }^{1}$.

Tregenza et al. suggest that, in most species, males will rapidly identify and attempt to mate only with those females with whom they are compatible, thus reducing the potential for sexual conflict to promote divergence. But even if the necessary 'indicators' of female compatibility should be readily available and the males smart enough to exploit them, in a polymorphic population different females will experience a variable number of matings. This will induce fitness differences and initiate runaway coevolution, resulting in genetic divergence of isolated populations. Although the model does not describe all the specific processes involved in sexual conflict, other weaknesses are not yet apparent.

\section{S. Gavrilets}

Department of Ecology and Evolutionary Biology,

Department of Mathematics, University of

Tennessee Knoxville, Tennessee 37996, USA

1. Gavrilets, S. Nature 403, 886-889 (2000).

2. Parker, G. A. \& Partridge, L. Phil. Trans. R. Soc. Lond. B 353, 261-274 (1998).

3. Arnqvist, G. \& Nilson, T. Anim. Behav. (in the press).

4. Fowler, K. \& Partridge, L. Nature 338, 760-761 (1989).

5. Gems, D. \& Riddle, D. L. Nature 379, 723-725 (1996).

6. Jennions, M. D. \& Petrie, M. Biol. Rev. 75, 21-64 (2000).

\section{Sandwich films \\ Properties of a new soft magnetic material}

he development of advanced electromagnetic devices has been constrained

by a lack of soft magnetic materials with a suitably high saturation magnetization (over 20 kilogauss) and a large permeability roll-off frequency (greater than 1 gigaherz). For example, magnetic hard-disk-drive technology is rapidly approaching the perceived superparamagnetic limit at which the stored bits become thermally unstable ${ }^{1}$ — disks with higher anisotropy are more stable but are not usable because magnetic write heads become saturated. Here we describe a new soft magnetic material with a saturation magnetization of 24 kilogauss and a large permeability of 1,000-1,400 in a wide frequency range of up to about 1.2 gigaherz. This new material promises to have wide application in devices such as magnetic recording heads and integrated inductors.

Soft magnetic materials such as $\mathrm{Co}_{0.57} \mathrm{Ni}_{0.13} \mathrm{Fe}_{0.30}$ (ref. 2) and FeAlN (ref. 3) have a saturation magnetization of about 20 $\mathrm{kG}$, and $\mathrm{Fe}_{1-x} \mathrm{Co}_{x}$ alloys (where $0.3 \leqslant x \leqslant 0.4$ ) have the highest saturation magnetization that occurs in nature (about $24.5 \mathrm{kG}$ ). However, the coercivity of these FeCo alloys tends to be greater than 5 oersteds, making them unsuitable for magnetic write heads 4 . Attempts to obtain soft magnetic materials have involved making artificial structures ${ }^{5}$.

We have created a sandwich structure, $\mathrm{Ni}_{0.81} \mathrm{Fe}_{0.19} /\left(\mathrm{Fe}_{0.7} \mathrm{Co}_{0.3}\right){ }_{0.95} \mathrm{~N}_{0.05} / \mathrm{Ni}_{0.81} \mathrm{Fe}_{0.19}$, in which the FeCoN film is $100 \mathrm{~nm}$ thick, and each $\mathrm{Ni}_{0.81} \mathrm{Fe}_{0.19}$ Permalloy layer is $5 \mathrm{~nm}$ thick, comprising only $4.5 \%$ of the volume of the sandwich. Typical hysteresis loops (magnetization, $M$, plotted against magnetic field, $H$ ) of the sandwich films, determined by using a vibrating sample magnetometer, are shown in Fig. 1, inset:

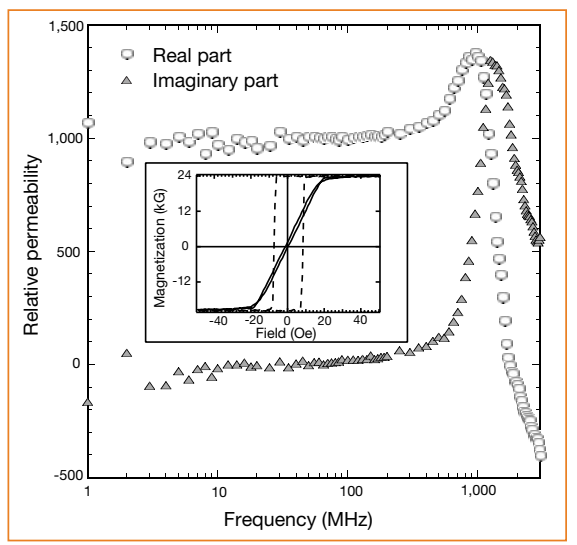

Figure 1 Relative permeability, both real and imaginary parts, versus frequency for a typical $\mathrm{Ni}_{0.81} \mathrm{Fe}_{0.19}(5 \mathrm{~nm}) /\left(\mathrm{Fe}_{0.7} \mathrm{Co}_{0.3}\right)_{0.95} \mathrm{~N}_{0.05}(100 \mathrm{~nm}) /$ $\mathrm{Ni}_{0.81} \mathrm{Fe}_{0.19}(5 \mathrm{~nm})$ film. Inset, typical $M-H$ loops of the sandwich film. Dashed line, easy axis loop; solid line, hard axis loop. 
coercivities of the sandwich films are 0.6 and 7.8 Oe in the hard and easy axis, respectively; the anisotropy field, $H_{\mathrm{k}}$, is about 20 Oe. Much thicker soft magnetic films with similar properties can be realized by laminating many $\mathrm{Ni}_{0.81} \mathrm{Fe}_{0.19} /$ $\left(\mathrm{Fe}_{0.7} \mathrm{Co}_{0.3}\right)_{0.95} \mathrm{~N}_{0.05}$ bilayers.

In contrast, coercivities of the $\mathrm{FeCoN}$ single layer are about 5 and 18 Oe in the hard and easy axis, respectively. A purely magnetostatic consideration would give the average coercivity of the sandwich as about $91 \%$ of that of the $\mathrm{FeCoN}$ single layer if the coercivities of Permalloy are negligible. Therefore, the observed reduction in coercivity due to sandwiching is surprisingly large. We believe that the reduction of magnetic anisotropy dispersion due to the Permalloy seed and applied deposition field (about 50 Oe) may be important here.

We also measured the hard-axis magnetic permeability, both real and imaginary parts, of the new material using a permeance meter with a frequency range up to $3 \mathrm{GHz}$ (ref. 6) (Fig. 1). The real permeability is $1,000-1,400$ at frequencies up to $\sim 1.2 \mathrm{GHz}$, and the imaginary permeability peaks at $\sim 1.5 \mathrm{GHz}$, which corresponds to the ferromagnetic resonance frequency, and is slightly lower than the calculated one: $f_{\mathrm{FMR}}=\gamma \sqrt{4 \pi M_{\mathrm{s}} \times H_{\mathrm{k}}}=1.9 \mathrm{GHz}$, where $\gamma=2.8 \mathrm{MHz}$ per oersted and is the gyromagnetic ratio, and $4 \pi M_{\mathrm{s}}$ is the saturation magnetization, all in gaussian units. The resistivity of the film is $\sim 50 \mu \Omega \mathrm{cm}$, and its cut-off frequency due to eddy currents is $\sim 8.6 \mathrm{GHz}$, so the eddy currents can be neglected.

The permeability spectra confirm that our new films are promising for use in extremely high-density magnetic write heads ${ }^{3}$ as well as in integrated inductors operating in the gigaherz range ${ }^{7}$. Other soft magnetic materials described for applications at gigaherz frequencies, such as $\mathrm{Co}_{0.443} \mathrm{Fe}_{0.191} \mathrm{Hf}_{0.145} \mathrm{O}_{0.221}$ (ref. 8) and $\mathrm{Fe}_{0.878} \mathrm{Cr}_{0.046} \mathrm{Ta}_{0.002} \mathrm{~N}_{0.074}$ (ref. 9), have a permeability of about 200 and a lower saturation magnetization.

S. X. Wang ${ }^{\star}$, N. X. Sun ${ }^{\star}$, M. Yamaguchi $\dagger$, S. Yabukami $†$

${ }^{*}$ Laboratory of Advanced Materials, Department of Materials Science and Engineering, Stanford

University, Stanford, California 94305-4045, USA

$\dagger$ Research Institute of Electrical Communication,

Tohoku University, Sendai 980-8577, Japan

e-mail: sxwang@ee.stanford.edu

1. Charap, S. H., Lu, P. L. \& He, Y. IEEE Trans. Magn. 33, 978-983 (1997).

2. Osaka, T. et al. Nature 392, 796-798 (1998).

3. Wang, S. X. \& Taratorin, A. M. Magnetic Information Storage Technology (Academic, Boston, 1999).

4. Sun, N. X. \&. Wang, S. X. IEEE Trans. Magn. (in the press).

5. Clow, H. Nature 194, 1035-1036 (1962).

6. Yabukami, S. et al. J. Appl. Phys. 85, 5148-5150 (1999).

7. Yamaguchi, M. et al. J. Appl. Phys. 85, 7919-7922 (1999).

8. Hayakawa, Y. et al. J. Appl. Phys. 81, 3747-3752 (1997).

9. Jin, S. et al. Appl. Phys. Lett. 70, 3161-3163 (1997).

NATURE | VOL 407 | 14 SEPTEMBER 2000 | www.nature.com

\section{Virology}

\section{KSHV-like herpesviruses} in chimps and gorillas

mong the herpesviruses ${ }^{1}$, KSHV (Kaposi's-sarcoma-associated herpesvirus) is the human prototype of the rhadinovirus genus ${ }^{2}$. Rhadinoviruses (or $\gamma_{2}$-herpesviruses) are found in several animal species, including New and Old World monkeys, but not in the great apes ${ }^{3-5}$. Here we describe the detection and sequencing of a polymerase gene fragment from three new rhadinoviruses discovered in chimpanzees and in a gorilla, which are more closely related to KSHV than to any other virus of this genus described so far. Our results indicate that the great apes from central Africa could provide a reservoir of new $\gamma_{2}$-herpesviruses that are potentially transmissible to humans.

As KSHV and Kaposi's sarcoma are endemic in central Africa, we tested for KSHV-related viruses in great apes from central Africa. Blood specimens were taken from 30 wild-born animals (25 chimpanzees, Pan troglodytes, and five gorillas, Gorilla gorilla) originating from Cameroon and Gabon ${ }^{6}$. An immunofluorescence assay that detects both latent and lytic KSHV antigens revealed a clear reactivity in the plasma of 20 out of 25 chimpanzees and two out of five gorillas at a 1 in 40 dilution ${ }^{7}$.

We amplified a fragment of DNA isolated from peripheral blood mononuclear cells taken from five of these animals (four chimpanzees and one gorilla) by using nest-

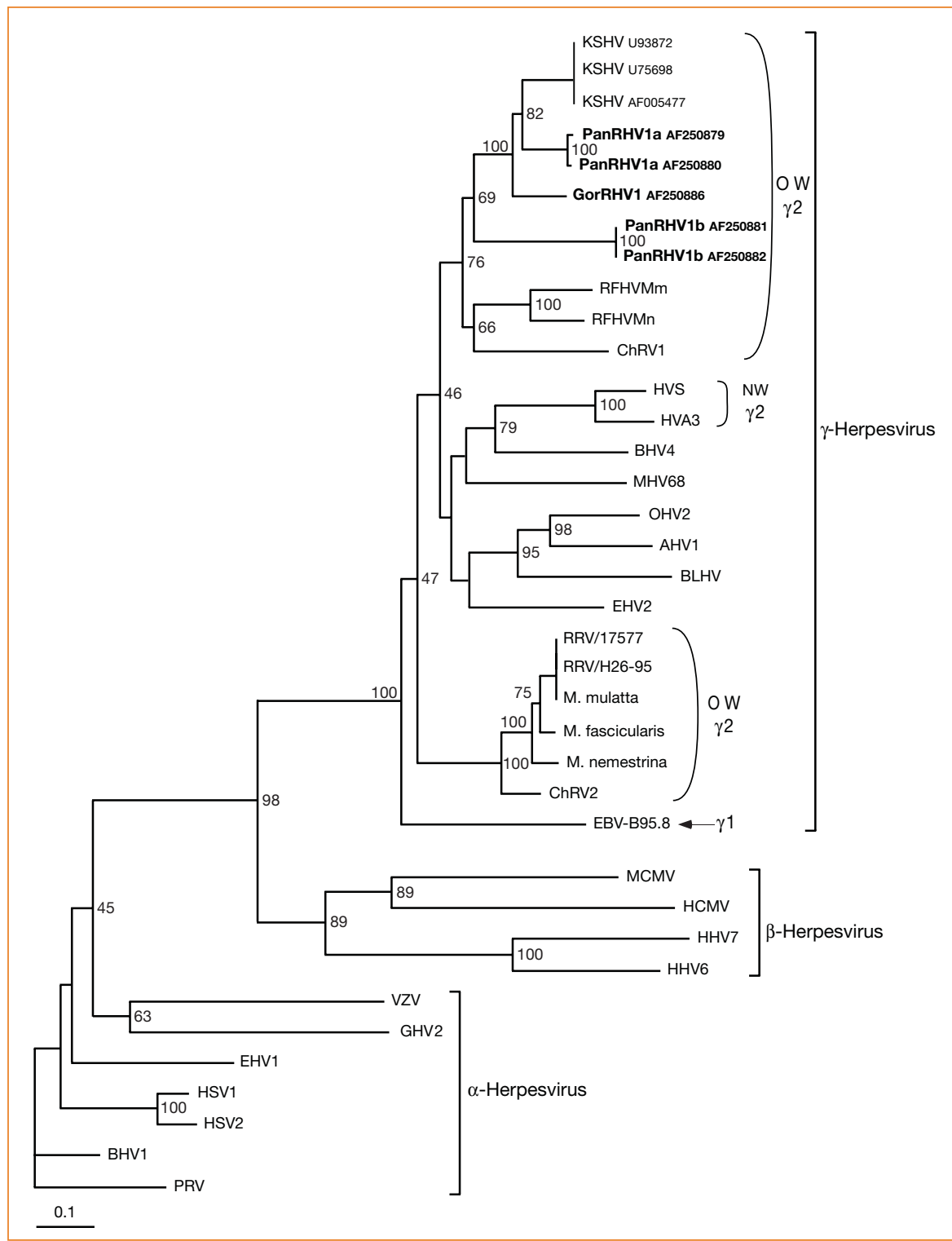

Figure 1 Phylogenetic tree resulting from analysis of 37 selected 454-base-pair fragments ${ }^{5}$ of the herpesvirus DNA-polymerase gene. Phylogeny was derived by using the neighbour-joining method applied to pairwise sequence distances calculated by the Kimura twoparameter method. Horizontal branch lengths are drawn to scale, with the bar indicating 0.1 nucleotide replacements per site. Numbers at each node indicate the percentage of bootstrap samples (out of 100) in which the cluster to the right is supported. Brackets on the right indicate previously defined subfamily and genus herpesviral classification. OW, Old World; NW, New World. 